\title{
Propagation of Supersymmetric Charged Sleptons at High Energies
}

\author{
M. H. Reno \\ Department of Physics and Astronomy, University of Iowa, Iowa City, Iowa 52242 USA \\ I. Sarcevic and S. Su \\ Department of Physics, University of Arizona, Tucson, Arizona 85721, USA
}

\begin{abstract}
The potential for neutrino telescopes to discover charged stau production in neutrino-nucleon interactions in Earth depends in part on the stau lifetime and range. In some supersymmetric scenarios, the next lightest supersymmetric particle is a stau with a decay length on the scale of $10 \mathrm{~km}$. We evaluate the electromagnetic energy loss as a function of energy and stau mass. The energy loss parameter $\beta$ scales as the inverse stau mass for the dominating electromagnetic processes, photonuclear and $e^{+} e^{-}$pair production. The range can be parameterized as a function of stau mass, initial energy and minimum final energy. In comparison to earlier estimates of the stau range, our results are as much as a factor of two larger, improving the potential for stau discovery in neutrino telescopes.
\end{abstract}

\section{INTRODUCTION}

Neutrino telescopes have great potential for probing physics beyond the Standard Model when new particles are created by high energy cosmic neutrino passing through the Earth. A recent proposal [1] showed that the detection of two nearly parallel charged particle tracks from a pair of metastable staus, which are produced as secondary particles in very high energy neutrino-nucleon interactions, may provide a unique way of probing the supersymmetry (SUSY) breaking scale in weak scale supersymmetry models.

Ultrahigh energy neutrinos from astrophysical sources travel undeflected to the Earth. They would then interact with the nucleons in the Earth and pair produce two superparticles via the $t$-channel chargino or neutralino exchanges: $\nu N \rightarrow \tilde{l} \tilde{q}$. In SUSY models where gravitino is the lightest supersymmetric particle (LSP) and stau is the next lightest supersymmetric particle (NLSP), heavier slepton and squarks instantly decay into the stau NLSP 2]. The high energy stau would then travel a long distance, typically more than tens to $10^{4}$ kilometers, before it decays into the gravitino LSP. The superparticle pair production rate, however, is very small in comparison to the standard model charged-current cross section. Thus production of staus from downward neutrinos is negligible compared to showers from production through the standard model processes. However, the observability of the stau produced via upward going neutrinos could be enhanced. The small stau production cross section are compensated by the enlarged effective volume of an instrumented region due to the very long lifetime of the stau and its relatively slow energy loss in the water, ice or rock surrounding the detector [1] .

The potential for neutrino telescopes to discover stau production in neutrino-nucleon interactions in Earth depends in part on the stau lifetime and range. In order to refine estimates of the signal from metastable staus, it is crucial to determine the energy loss of the high energy stau as it traverses the Earth. The average energy loss of a particle traversing a distance $X$ is given by

$$
-\left\langle\frac{d E}{d X}\right\rangle=\alpha+\beta E
$$

where $E$ is the energy of the particle, $\alpha$ describes ionization energy loss, and $\beta$ is the radiative energy loss. Ref. 1] estimated the stau range using Eq. (11) with the approximation that both $\alpha$ and $\beta$ are constants with respect to energy. In this paper, we evaluate both the energy and mass dependence of the stau electromagnetic energy loss. Our results are applicable to any metastable heavy charged slepton. We determine $\beta$ and we evaluate the average stau range using a one-dimensional Monte Carlo simulation of stau propagation in rock. We comment on the implications of the new results for the evaluation of event rates by Albuquerque, Burdman and Chacko in Ref. [1].

This paper is organized as follows. In Sec. [II we analyzed in detail the stau energy loss through photonuclear scattering, pair production and bremsstrahlung. In Sec. III] we show the stau range using both a onedimensional Monte Carlo evaluation and a parametrized approximate formula. In Sec. IV we conclude.

\section{STAU ENERGY LOSS}

The radiative energy loss $\beta$ receives contributions from bremsstrahlung, pair production and photonuclear scattering:

$$
\beta^{i}(E)=\frac{N}{A} \int_{y_{\min }}^{y_{\max }} d y y \frac{d \sigma^{i}(y, E)}{d y},
$$

where $y$ is the fraction of stau energy loss in the radiative interaction:

$$
y=\frac{E-E^{\prime}}{E}
$$

for final stau energy $E^{\prime}$. The superscript $i$ denotes bremsstrahlung (brem), pair production (pair) and pho- 


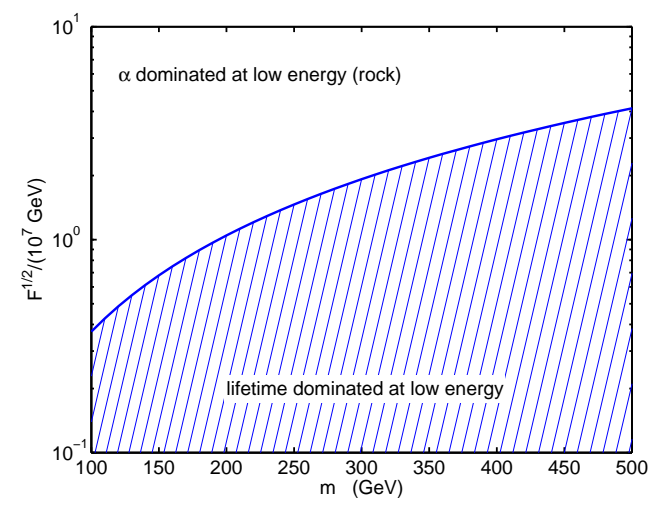

FIG. 1: Parameter space in $\left(m_{\tilde{l}}, \sqrt{F}\right)$ in which the stau range is dominated by either ionization energy loss or the lifetime, when $\beta E \ll \alpha$.

tonuclear (nuc) processes. Avogadro's number is $N$ and the atomic mass number of the target nucleus is $A$.

As we show below, the value for $\beta$ decreases with increasing stau mass. The ionization energy loss parameter $\alpha$ is nearly constant as a function of mass of the particle, namely [3]

$$
\alpha \simeq 2 \times 10^{-3} \mathrm{GeV} \mathrm{cm}^{2} / \mathrm{g} .
$$

For low enough energies, where $\beta E \ll \alpha$ (for $\beta \sim 5 \times 10^{-9}$ $\mathrm{cm}^{2} / \mathrm{g} \cdot\left(150 \mathrm{GeV} / m_{\tilde{l}}\right)$, it corresponds to $E \ll 4 \times 10^{5}$ $\left.\mathrm{GeV} \cdot\left(m_{\tilde{l}} / 150 \mathrm{GeV}\right)\right)$, either the lifetime or ionization energy loss determines the stau range, which scales linearly with energy. This is shown in Fig. [1] These two regions are separated by the line which corresponds to $c \tau \rho=m_{\tilde{l}} / \alpha$, where $m_{\tilde{l}}$ is the stau mass, $\rho$ is the density, taken to be the density of standard rock $\rho=2.65$ $\mathrm{g} / \mathrm{cm}^{3}$, and

$$
c \tau=\left(\frac{\sqrt{F}}{10^{7} \mathrm{GeV}}\right)^{4}\left(\frac{100 \mathrm{GeV}}{m_{\tilde{l}}}\right)^{5} 10 \mathrm{~km}
$$

is the stau lifetime. The quantity $\sqrt{F}$ is the supersymmetry breaking scale. In low energy SUSY breaking models, the SUSY breaking scale $\sqrt{F}$ could be as low as $10^{7}$ $\mathrm{GeV}$ [2], leading to a stau decay length of $10 \mathrm{~km}$ or longer.

\section{A. Stau Energy Loss due to Photonuclear Interactions}

We obtain a photonuclear cross section that describes the interactions of charged staus $(\tilde{\tau}$, or more generically charged sleptons $\tilde{l})$ with nuclei via virtual photon exchange. Our approach [4] is to treat the $\tilde{l} N \rightarrow \tilde{l} X$ using the deep-inelastic scattering formalism [5] in terms of the two nucleon electromagnetic structure functions $F_{1}$ and $F_{2}$.

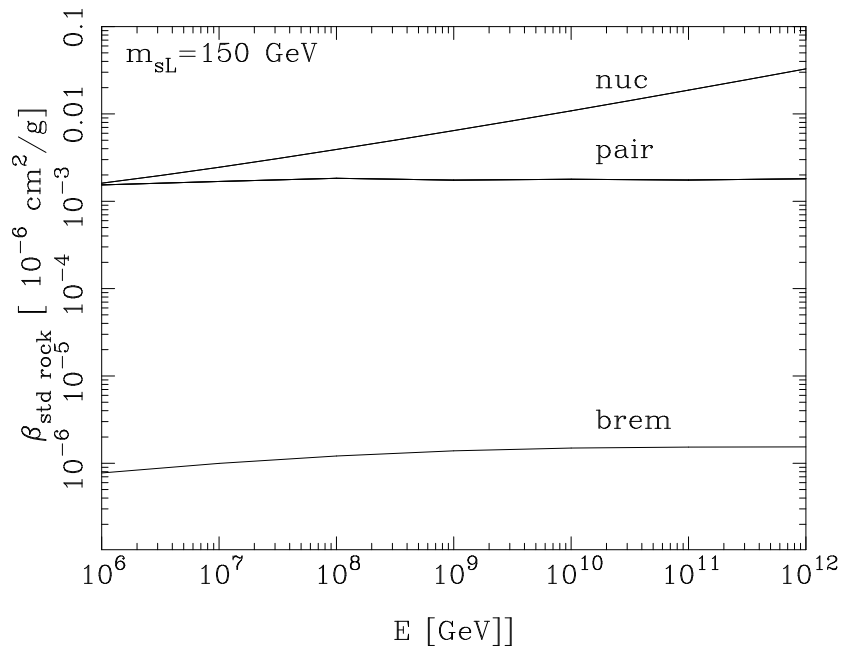

FIG. 2: Energy loss of stau due to photonuclear (nuc) interactions, pair production (pair) and bremsstrahlung (brem) for a stau mass of $150 \mathrm{GeV}$.

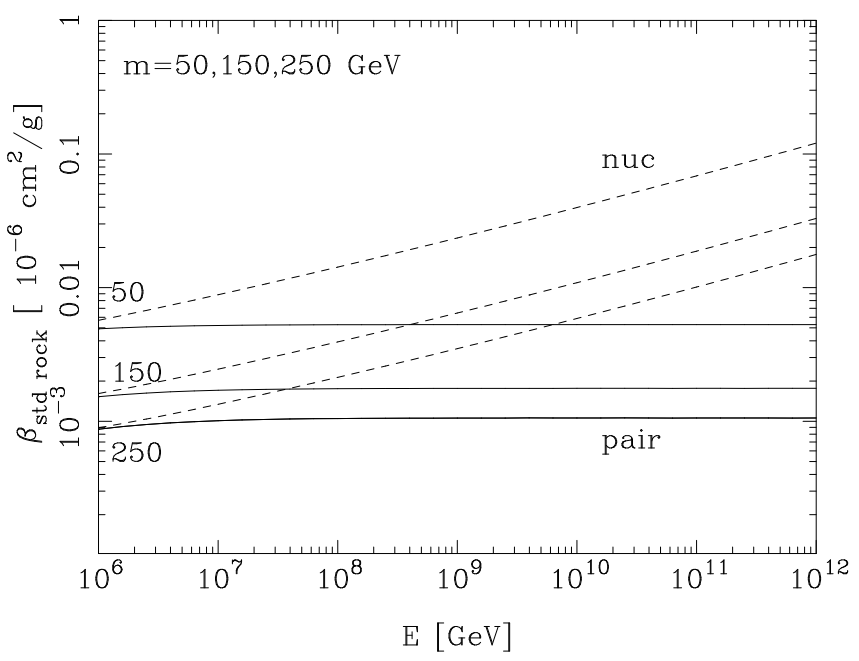

FIG. 3: Energy loss of stau due to photonuclear and pair production for $m_{\tilde{\tau}}=50 \mathrm{GeV}, m_{\tilde{\tau}}=150 \mathrm{GeV}$ and $m_{\tilde{\tau}}=250$ $\mathrm{GeV}$.

We use $F_{2}$ parameterized by Abramowitz, Levin, Levy and Maor (ALLM) [6] to fit HERA data for a wide range of $Q^{2}$ and $x$. The ALLM parameterization of the structure function is based on the Regge approach and consists of a Pomeron and a Reggeon contribution [6]. Other approaches [7], which focus on the diffractive region, but also include a perturbative component, give qualitatively similar results for the photonuclear energy loss of leptons.

For stau scattering with nucleons, $\tilde{\tau}(k) N(p) \rightarrow$ $\tilde{\tau}\left(k^{\prime}\right) X$, the differential cross section is given by

$$
\frac{d^{2} \sigma\left(x, Q^{2}\right)}{d Q^{2} d x}=\frac{4 \pi \alpha^{2}}{Q^{4}} \frac{F_{2}\left(x, Q^{2}\right)}{x}\left[1-y+\frac{y^{2}}{4}\right.
$$




$$
\left.-\left(1+\frac{4 m_{\tilde{l}}^{2}}{Q^{2}}\right) \frac{y^{2}\left(1+4 M^{2} x^{2} / Q^{2}\right)}{4\left(1+R\left(x, Q^{2}\right)\right)}\right],
$$

where $-Q^{2}=q^{2}=\left(k-k^{\prime}\right)^{2}, x=Q^{2} /(2 p \cdot q), y$ as in Eq. (3), $m_{\tilde{l}}$ is the stau mass, and $M$ is the nucleon mass. $R\left(x, Q^{2}\right)$ parametrizes the violation of the Callan-Gross relation $2 x F_{1}\left(x, Q^{2}\right)=F_{2}\left(x, Q^{2}\right)$ :

$$
R\left(x, Q^{2}\right)=\frac{\left(1+\frac{4 M^{2} x^{2}}{Q^{2}}\right) F_{2}\left(x, Q^{2}\right)-2 x F_{1}\left(x, Q^{2}\right)}{2 x F_{1}\left(x, Q^{2}\right)} .
$$

Since $R\left(x, Q^{2}\right)$ is small [8], we approximate $R=0$.

To obtain $\beta$, we convert the differential cross section to a dependence on $y$ and $Q^{2}$ :

$$
\frac{d^{2} \sigma\left(y, Q^{2}\right)}{d Q^{2} d y}=\frac{x}{y} \frac{d^{2} \sigma\left(x, Q^{2}\right)}{d Q^{2} d x} .
$$

We use the following limits of integration:

$$
\begin{aligned}
Q_{\min }^{2} & \leq Q^{2} \leq 2 M E y-\left(\left(M+m_{\pi}\right)^{2}-M^{2}\right) \\
y_{\min } & \leq y \leq 1-m_{\tilde{l}} / E
\end{aligned}
$$

where $Q_{\min }^{2} \simeq m_{\tilde{l}}^{2} y^{2} /(1-y)$ and $y_{\min } \simeq\left(\left(M+m_{\pi}\right)^{2}-\right.$ $\left.M^{2}\right) /(2 M E)$.

For comparison, the photonuclear cross section for the standard model partner, the charged lepton, is given by [4]

$$
\begin{aligned}
\frac{d^{2} \sigma\left(x, Q^{2}\right)}{d Q^{2} d x}= & \frac{4 \pi \alpha^{2}}{Q^{4}} \frac{F_{2}\left(x, Q^{2}\right)}{x}\left[1-y-\frac{M x y}{2 E}\right. \\
& \left.+\left(1-\frac{2 m_{l}^{2}}{Q^{2}}\right) \frac{y^{2}\left(1+4 M^{2} x^{2} / Q^{2}\right)}{2}\right]
\end{aligned}
$$

in the $R \simeq 0$ limit. At high energies, the scalar and fermion evaluation of $\beta^{\text {nuc }}$ give the same numerical values for equal scalar and fermion masses because the energy loss is dominated by the small $y$ region of integration.

Our result for $\beta^{\text {nuc }}$ for $\tilde{\tau}$ 's with a mass of $150 \mathrm{GeV}$ is given by the upper curve in Fig. 22 For $m_{\tilde{\tau}}=150 \mathrm{GeV}$, it is increasing with energy from $\sim 2 \times 10^{-9} \mathrm{~cm}^{2} / \mathrm{g}$ at $10^{6} \mathrm{GeV}$ to $3 \times 10^{-8} \mathrm{~cm}^{2} / \mathrm{g}$ at $10^{12} \mathrm{GeV}$.

The dashed curves in Fig. 3] represent the photonuclear contribution to $\beta$ for three stau masses: $50 \mathrm{GeV}$, $150 \mathrm{GeV}$ and $250 \mathrm{GeV}$, showing that $\beta$ has a $1 / m_{\tilde{l}}$ dependence on the stau mass. This can be understood based on the argument which has been discussed in the context of electron-positron production in muon scattering by Tannenbaum in Ref. [9]. Qualitatively,

$$
F_{2} \sim \frac{Q^{2}}{Q^{2}+m_{0}^{2}} f_{2}
$$

where to first approximation, $f_{2}$ is constant for small $y$. The parameter $m_{0}^{2}$ is $0.32 \mathrm{GeV}^{2}$ for the Abramowitz et al. parameterization [6]. We can approximate

$$
\beta \simeq \int d y \int_{Q_{\min }^{2}}^{Q_{\max }^{2}} d Q^{2} \frac{4 \pi \alpha^{2}}{Q^{2}\left(Q^{2}+m_{0}^{2}\right)} f_{2} .
$$

The integral over $Q^{2}$ is approximately independent of $Q_{\max }^{2}$ and depends on $Q_{\min }^{2} / m_{0}^{2}$. In the small $y$ limit, $Q_{\min }^{2} \simeq m_{\tilde{l}}^{2} y^{2}$. The change of variables of the integral from $y$ to $z$ where

$$
z=\frac{m_{\tilde{l}} y}{m_{0}}
$$

yields a Jacobian factor of $m_{0} / m_{\tilde{l}}$ multiplying the integral. The stau mass dependence of $\beta$ is given by this Jacobian factor.

\section{B. Stau Energy Loss due to Pair Production}

Next we consider $e^{+} e^{-}$pair production in collisions of staus with nuclei. In this section, we show that the dominant contribution to $\beta$ for the production of an $e^{+} e^{-}$ pair by a scalar charged particle interaction with a bare nucleus is the same, at high energies, as for the fermion case. We then use the results of Kokoulin and Petrukhin for fermion scattering [10] which include all of the diagrams and incorporate a smooth transition between the unscreened to screened nucleus.

This process is a very important contribution to the energy loss of muons and taus. In the case of staus, it is also important. There are several diagrams that contribute. They are shown in Fig. [4

The diagrams can be classified into two categories. Diagrams (a1) and (a2) are "two photon" contributions to $e^{+} e^{-}$pair production. Diagrams (b1), (b2) and (c) combine to make the gauge invariant set of "virtual bremsstrahlung" contributions to $e^{+} e^{-}$pair production. For taus instead of staus, the virtual bremsstrahlung graphs are of the type (b1) and (b2). The interference term between two photon (charge conjugation even) and virtual bremsstrahlung (C-odd) contributions vanishes in the integral.

At relativistic energies, the two photon contribution dominates because of the presence of two quasi-real photons. This is independent of the spin of the charged particle (tau or stau) that emits one quasi-real photon. The two photon process leads to a factor proportional to the square of the classical electron radius $r_{e}=e^{2} / m_{e} c^{2}$, while the virtual bremsstrahlung contribution is proportional to the square of the classical heavy lepton radius [11], a result that carries over to the heavy slepton case at high energy. The dominance of the two photon process was first demonstrated in Ref. [12] and has been studied in many contexts, including lepton pair production in colliding beam experiments [13], and more recently in heavy ion collisions [14].

Since the two photon diagrams dominate, we calculate only these two contributions. The cross section corresponding to these diagrams, in the notation of Kelner [15], is given by:

$$
d \sigma=\frac{Z^{2} \alpha^{4}}{\pi^{4}}\left[\left(p_{1} \cdot u\right)^{2}-m_{\tilde{l}}^{2}\right]^{-1 / 2} \frac{1}{k_{1}^{4} Q^{4}} A^{\alpha \beta} B_{\alpha \beta}^{\mu \nu} u_{\mu} u_{\nu}
$$




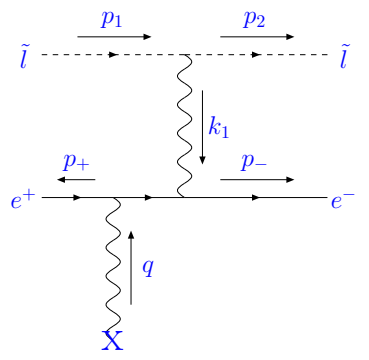

(a1)

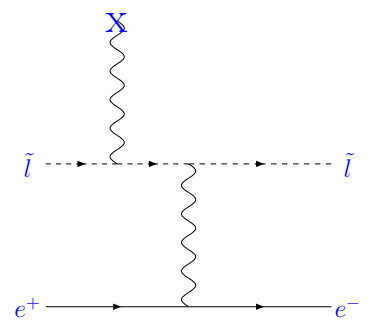

(b1)

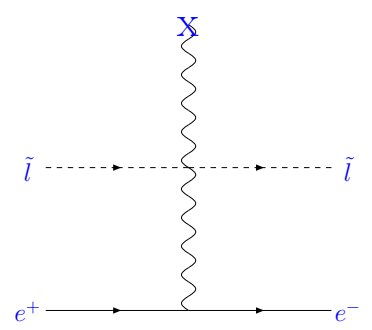

(c)

FIG. 4: Feynman diagrams for $e^{+} e^{-}$pair production by stau scattering from a nucleus with nuclear momentum transfer $q$. Diagrams (a1) and (a2) dominate $\beta^{\text {pair }}$.

$$
\cdot \frac{d \mathbf{p}_{+}}{p_{+}^{0}} \frac{d \mathbf{p}_{-}}{p_{-}^{0}} \frac{d \mathbf{p}_{2}}{p_{2}^{0}} \frac{d \mathbf{q}}{u_{0}} \delta\left(q+k_{1}-p_{+}-p_{-}\right)
$$

where $u_{\mu}$ is the velocity of the nucleus, and $p_{1}, p_{2}, p_{+}$ and $p_{-}$are the momentum for incoming stau, outgoing stau, (outgoing) position and electron, respectively. In addition, $k_{1}=p_{1}-p_{2}, q=p_{+}+p_{-}-k_{1}$, and $Q^{2}=-q^{2}$. For scalar interactions,

$$
A^{\alpha \beta}=\left(p_{1}+p_{2}\right)^{\alpha}\left(p_{1}+p_{2}\right)^{\beta} .
$$

The quantity $B_{\alpha \beta}^{\mu \nu}$ is the usual fermion trace involving the sum of the two diagrams with the two photon vertices. A series of separations and integrals can be performed so that the differential cross section can be written in the following form [17]:

$$
\begin{aligned}
d \sigma= & \frac{2}{3} \frac{Z^{2} \alpha^{4}}{\pi m_{e}^{2}} I_{1}(1-y) d x_{+} \frac{d \omega}{\omega} d Q^{2} \\
& \cdot G\left(x_{+}, x_{-}, Q^{2}, y\right)
\end{aligned}
$$

where

$$
x_{+}=\frac{p_{+}^{0}}{\omega}, \quad x_{-}=\frac{p_{-}^{0}}{\omega}
$$

$$
\begin{aligned}
\omega & =p_{+}^{0}+p_{-}^{0} \\
y & =\frac{\omega}{p_{1}^{0}} \\
I_{1} & =\log \left[\frac{4 \omega^{2} x_{+}^{2} x_{-}^{2}}{m_{e}^{2} \gamma}\right]-1 \\
\gamma & =1+\frac{Q^{2}}{m_{e}^{2}} x_{+} x_{-} .
\end{aligned}
$$

One finds that $G=G\left(x_{+}, x_{-}, Q^{2}, y\right)$ is

$$
\begin{aligned}
G= & a_{1}\left(\frac{1}{Q^{2}}-\frac{x_{+} x_{-}}{\gamma m_{e}^{2}}\right)-b_{1} \frac{\xi m_{e}^{2}}{Q^{4} x_{+} x_{-}} \\
& -c_{1} \frac{x_{+} x_{-}}{m_{e}^{2} \gamma^{2}} \\
a_{1}= & \left(2 x_{+}^{2}+2 x_{-}^{2}+1\right)(D+\lambda C) \\
& +2 D \xi\left(x_{+}^{2}+x_{-}^{2}+1\right) \\
b_{1}= & D\left(2 x_{+}^{2}+2 x_{-}^{2}+1\right) \\
c_{1}= & D(1+\xi)+\lambda C \\
& +2 x_{+} x_{-}[\lambda C-(2+\lambda) D]
\end{aligned}
$$

with the additional definitions

$$
\begin{aligned}
\xi & =\frac{m_{\tilde{l}}^{2}}{m_{e}^{2}} x_{+} x_{-} 2 \lambda \\
\lambda & =\frac{y^{2}}{2(1-y)} .
\end{aligned}
$$

The quantities $C$ and $D$ [17] characterize the incident particle as scalar or fermion:

$$
\begin{array}{lll}
C=0 & D=1 & \text { scalar case }, \\
C=1 & D=1 & \text { fermion case } .
\end{array}
$$

The resulting $\beta$ from diagrams (a1) and (a2) of Fig. 4 yielding Eq. (18), is obtained for the scalar case and found to be the same as for the fermion case to within the numerical accuracy of our integration using the fortran program VEGAS [16] (here, a relative error of $10^{-4}$ ). This can be understood explicitly from Eq. (18), where each factor of $\mathrm{C}$, which differs for scalar and fermion cases, is multiplied by $\lambda$. The quantity $\beta$ is dominated by small $y$, so $\lambda \ll 1$. On a more qualitative level, one notes that the Gordon decomposition of the fermion current has a term proportional to $\left(p_{1}+p_{2}\right)_{\mu}$, the scalar coupling, which dominates in the high energy limit.

Because the two photon ((a1) and (a2) diagrams) process gives $\beta^{\text {pair }}$, and at high energies, the difference between fermion and scalar scattering to produce the $e^{+} e^{-}$ pair is negligible, in the results we show below we have used the complete fermion expression of Ref. [10] with the substitution $m_{\mu} \rightarrow m_{\tilde{l}}$ to describe $\beta^{\text {pair }}$ for staus at high energy.

The results for $\beta^{\text {pair }}$ are shown by the labeled curve in Fig. 2 for $m_{\tilde{l}}=150 \mathrm{GeV}$. The scaling with mass is shown in Fig. 3. where we show $\beta^{\text {pair }}$ with solid lines for 


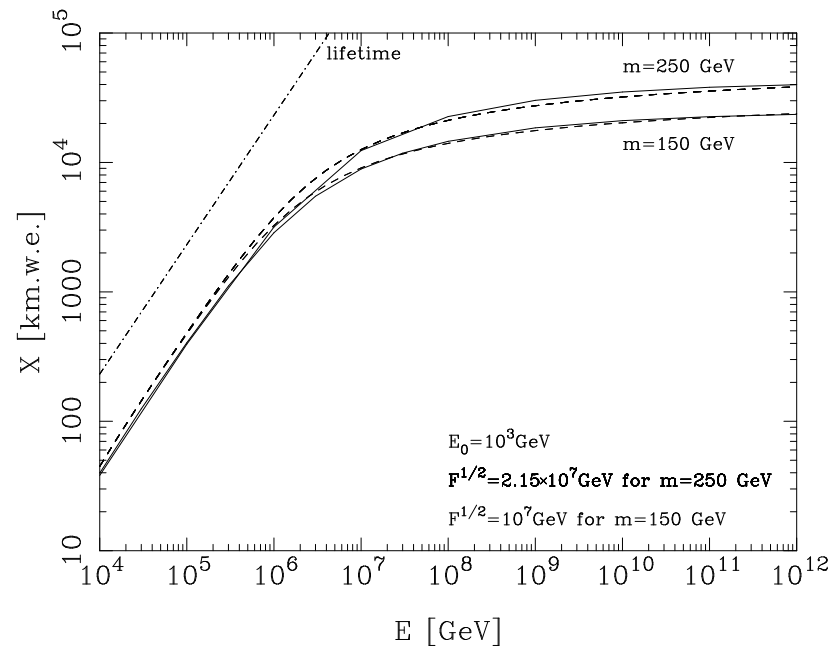

FIG. 5: Stau range (solid) and time dilated decay length (dotdashed line) for different values of $\sqrt{F}$ and for $m_{\tilde{l}}=150 \mathrm{GeV}$ and $m_{\tilde{l}}=250 \mathrm{GeV}$. The values of $\sqrt{F}$ are chosen to make the lifetimes equal. The dashed line shows the parameterization of the range using Eq. (24-25).

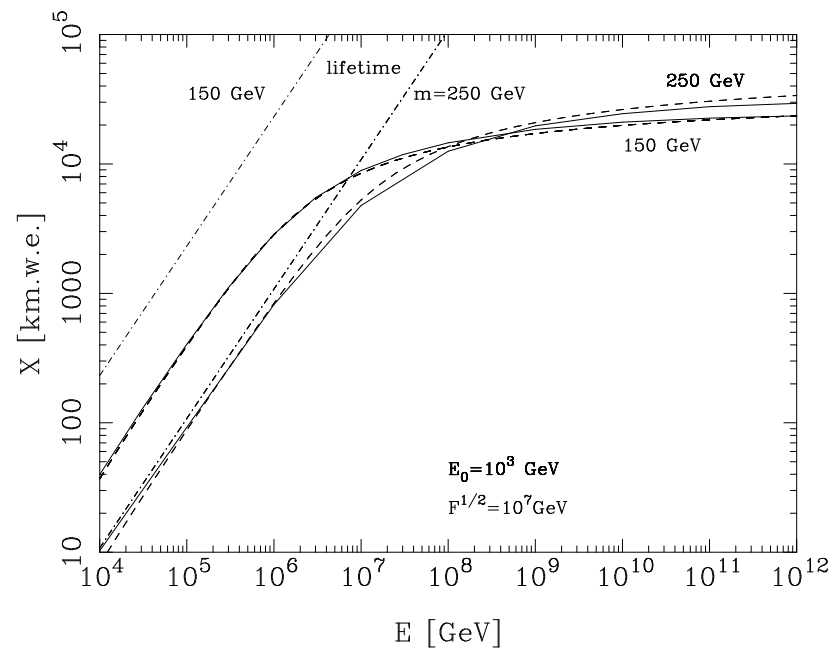

FIG. 6: Stau range (solid) and time dilated decay length (dotdashed line) for $m_{\tilde{l}}=150 \mathrm{GeV}$ and $m_{\tilde{l}}=250 \mathrm{GeV}$ for $\sqrt{F}=$ $10^{7} \mathrm{GeV}$. The dashed line shows the parameterization of the range using Eq. (24-26).

$m_{\tilde{l}}=50,150$ and $250 \mathrm{GeV}$. Again, the $1 / m_{\tilde{l}}$ behavior of $\beta$ appears. As noted in Ref. 9], the differential cross section (in $y$ and $Q^{2}$ ) can be approximated in the small $y$ limit by a form similar to Eq. (12), where instead $f_{2} \rightarrow$ $\sigma_{e^{+} e^{-}}$, the photopair production cross section which is nearly constant, and $m_{0} \rightarrow 4 m_{e}$ related to the threshold for electron-positron pair production. The same Jacobian factor, now $\sim m_{e} / m_{\tilde{l}}$ determines the $m_{\tilde{l}}$ dependence of $\beta^{\text {pair }}$

\section{Stau Energy Loss due to Bremsstrahlung}

The bremsstrahlung contribution to $\beta$ is much smaller than the pair production and photonuclear contributions by an additional factor of $1 / m_{\tilde{l}}$. A textbook discussion of $\beta^{\text {brem }}$ [18] shows that

$$
\frac{d \sigma}{d y} \propto \frac{\bar{\sigma}}{y}
$$

where $\bar{\sigma}=Z^{2} \alpha^{3} / m_{\tilde{l}}^{2}$. The expression for $\beta^{\text {brem }}$ therefore has a dependence on the particle mass through $\bar{\sigma}$, namely $1 / m_{\tilde{l}}^{2}$. This is explicitly shown in Fig. 2 for $m_{\tilde{l}}=150$ $\mathrm{GeV}$, where the evaluation of $\beta$ follows from Ref. [19].

\section{STAU RANGE}

Our evaluation of the stau range follows the same procedure for tau propagation outlined in Ref. [4]. Briefly, we perform a one-dimensional Monte Carlo evaluation which treats the first two terms in the energy loss

$$
-\frac{d E}{d X}=\alpha+\frac{N}{A} E \int_{0}^{y_{c u t}} d y y \frac{d \sigma}{d y}+\frac{N}{A} E \int_{y_{c u t}}^{1} d y y \frac{d \sigma}{d y},
$$

continuously, and the third term stochastically. We use $y_{c u t}=10^{-3}$. We evaluate the survival probability $P\left(E, E_{0}, z\right)$ as a function of initial energy $E$, minimum final energy $E_{0}$ and depth $z$. The average range is

$$
X\left(E, E_{0}\right)=\int d z P\left(E, E_{0}, z\right) .
$$

We note that the average range is not the same as the range of average energy loss, the solution to Eq. (11) 20]. Discussed below is a parameterization of an effective $\beta$ that, when substituted into the solution to Eq. (1), gives the approximate average range.

The average ranges of the $\tilde{\tau}$ for two masses, $m_{\tilde{l}}=150$ $\mathrm{GeV}$ and $m_{\tilde{l}}=250 \mathrm{GeV}$ are shown in Fig. 5 by the solid lines. For this figure and Fig. [6] the stau is propagated until its energy is reduced to $E_{0}=10^{3} \mathrm{GeV}$. Shown in Fig. [5 are the results for $\sqrt{F}=10^{7} \mathrm{GeV}$ for $m_{\tilde{l}}=150$ $\mathrm{GeV}$ and $\sqrt{F}=2.15 \times 10^{7} \mathrm{GeV}$ for the larger mass. The two values of $F$ are chosen to give the same lifetimes for $m_{\tilde{l}}=150$ and $250 \mathrm{GeV}$. The time dilated decay length is long compared to the distance over which energy is lost by the stau. This is clear in that we can parameterize the range without reference to the decay length. A convenient choice is to use the solution to Eq. (11) for the range of average loss with a constant $\beta$, but to use in the solution an energy dependent $\beta$. In terms of initial stau energy $E$ and final stau energy $E_{0}$, we write

$$
\begin{aligned}
X\left(E, E_{0}\right)= & \frac{1}{\beta} \ln \left[(\alpha+\beta E) /\left(\alpha+\beta E_{0}\right)\right] \\
\beta \simeq & \beta_{0}+\beta_{1} \ln \left(E / 10^{10} \mathrm{GeV}\right) \\
& +\beta_{2} \ln \left(E_{0} / 10^{3} \mathrm{GeV}\right)
\end{aligned}
$$




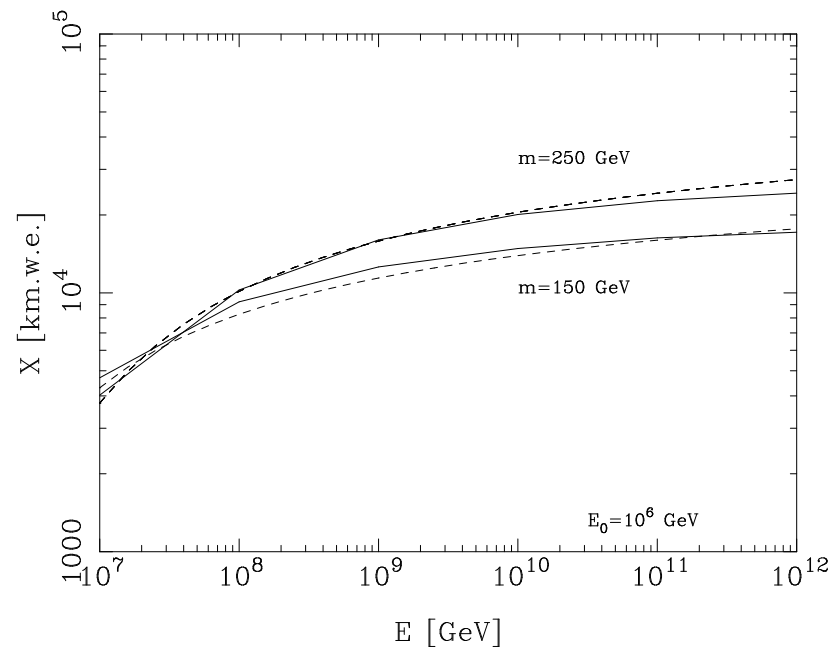

FIG. 7: Stau range (solid) for $\sqrt{F}=10^{7} \mathrm{GeV}$ and for $m_{\tilde{l}}=$ $150 \mathrm{GeV}$ and $m_{\tilde{l}}=250 \mathrm{GeV}$, with $E_{0}=10^{6} \mathrm{GeV}$. The dashed lines show the parameterization of the range following Eqs. (24-26) and the solid lines show the Monte Carlo results.

with

$$
\begin{aligned}
\alpha & =2 \times 10^{-3} \mathrm{GeV} \mathrm{cm}^{2} / \mathrm{g} \\
\beta_{0} & =5 \times 10^{-9} \mathrm{~cm}^{2} / \mathrm{g}\left(150 \mathrm{GeV} / m_{\tilde{l}}\right) \\
\beta_{1} & =2.8 \times 10^{-10} \mathrm{~cm}^{2} / \mathrm{g}\left(150 \mathrm{GeV} / m_{\tilde{l}}\right) \\
\beta_{2} & =2 \times 10^{-10} \mathrm{~cm}^{2} / \mathrm{g}\left(150 \mathrm{GeV} / m_{\tilde{l}}\right) .
\end{aligned}
$$

These parameters are obtained from a fit to the average range determined by the one-dimensional Monte Carlo program. In Fig. 5] the dashed lines following the solid curves show the parameterized fit, Eqs. (24) and (25), with $E_{0}=10^{3} \mathrm{GeV}$.

The average range of stau varies roughly linearly with stau energy $E$ for lower energy $E \leq 10^{6} \mathrm{GeV}$ in Fig. 5. For $m_{\tilde{l}}=150 \mathrm{GeV}$ and $\sqrt{F}=10^{7} \mathrm{GeV}$, the range is about $40 \mathrm{~km}$ water equivalence (w.e.) for $E=10^{4}$ $\mathrm{GeV}$, and about $3 \times 10^{3} \mathrm{~km}$ w.e. for $E=10^{6} \mathrm{GeV}$. This behavior can be obtained from Eq. (24) where for $\beta E \ll$ $\alpha, X \propto\left(E-E_{0}\right) / \alpha$. For higher energy, the average range varies logarithmically with energy: $X \propto \log \left(E / E_{0}\right) / \beta$, roughly a few $\times 10^{4} \mathrm{~km}$ w.e.

The average range at low energy has little mass dependence since $\alpha$ is a nearly a constant as a function of mass. At higher energy, the larger the mass of the particle, the longer the average range due to the $1 / m_{\tilde{l}}$ dependence of $\beta$.

For $\sqrt{F}$ fixed at $\sqrt{F}=10^{7} \mathrm{GeV}$, in the case of $m_{\tilde{l}}=150 \mathrm{GeV}$, the low energy range is dominated by ionization energy loss, while for $m_{\tilde{l}}=250 \mathrm{GeV}$, it is dominated by the decay length (see Fig. 6). For the latter case, the average range at low energy still has the linear dependence on $E$ since $\gamma c \tau=\left(E / m_{\tilde{l}}\right) c \tau$. A modification of Eq. (24) to account for the lifetime involves the substitution

$$
\alpha \rightarrow \alpha_{c}=\alpha+\frac{m_{\tilde{l}}}{c \tau \rho} .
$$

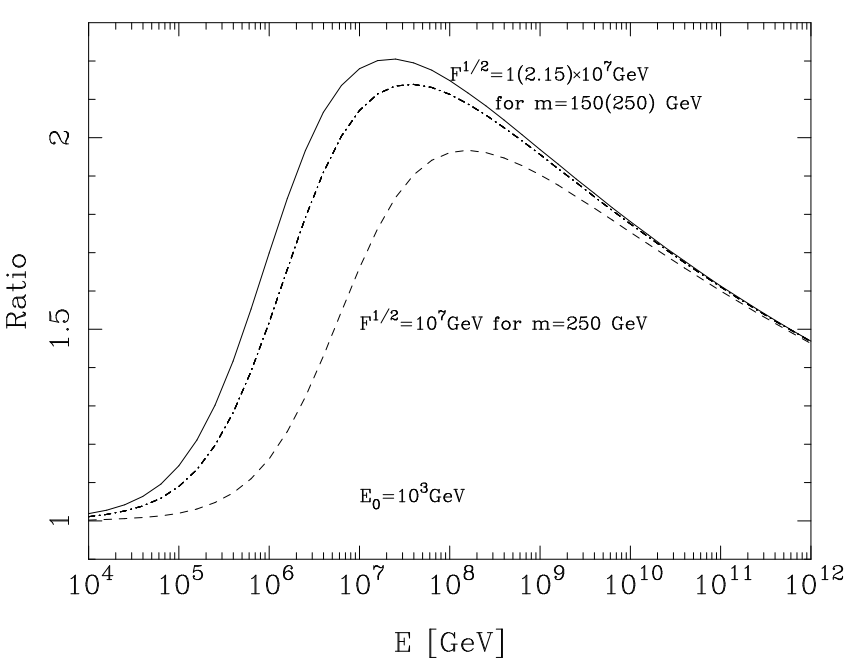

FIG. 8: Ratio of stau range using the parameterization of Eqs. (24-26) to stau range using a constant $\beta=0.8 \times 10^{-6} m_{\tau} / m_{\tilde{l}}$ $\mathrm{cm}^{2} / \mathrm{g}$ for $\sqrt{F}=10^{7} \mathrm{GeV}$ and $m_{\tilde{l}}=150 \mathrm{GeV}$ (solid) and $m_{\tilde{l}}=250 \mathrm{GeV}$ (dashed line), and for $\sqrt{F}=2.15 \times 10^{7} \mathrm{GeV}$ with $m_{\tilde{l}}=250 \mathrm{GeV}$ (dot-dashed line), for $E_{0}=10^{3} \mathrm{GeV}$.

With Eq. (24) and the energy dependent $\beta$, the substitution of $\alpha \rightarrow \alpha_{c}$ leads to a reasonable approximation to the range, as seen in Fig. 6] Numerically, for the three range curves shown in Figs. 5 and 6 the parameterization agrees to within $\sim 20 \%$ over the whole energy range. In fact, for the curves in Fig. 5 the ratio of parametrization of the range to the Monte Carlo evaluation of the range deviates from 1 only by $-8 \%-+1 \%$ from $10^{4}-10^{12}$ $\mathrm{GeV}$ initial stau energy. For the shorter lifetime shown in Fig. 6] the ratio of the parameterization to the Monte Carlo range deviates from 1 by $-5 \%-+15 \%$ except for $10^{4}-10^{5} \mathrm{GeV}$, where the deviation reaches as much as $-22 \%$.

At higher energy thresholds $E_{0}$, the effective $\beta$ increases, requiring the $\beta_{2}$ term in Eq. (25). In Fig. 7 we show the stau range and its parameterization for $E_{0}=10^{6} \mathrm{GeV}$. The average range is shorter than the case of $E_{0}=10^{3} \mathrm{GeV}$ due to the higher energy threshold. The ratio of the parameterization of the range to the Monte Carlo evaluation goes between 1.03- 0.88 for $m_{\tilde{l}}=150 \mathrm{GeV}$, and between $1.11-0.93$ for $m_{\tilde{l}}=250$ $\mathrm{GeV}$.

\section{DISCUSSION}

Albuquerque, Chacko and Burdman proposed to look for evidence of supersymmetry in a class of models by looking for pair-produced staus with nearly parallel tracks [1]. They concluded that there is a potential sensitivity of neutrino telescopes to a range of parameter space in certain low energy SUSY breaking models. Their evaluation of event rates of upward-going staus depends in part on the effective area of the detector and the range 
of the staus.

To first approximation, the energy loss of the stau can be characterized by constant $\alpha$ and $\beta_{\tilde{\tau}, c}$. This is what was done in Ref. [1], where $\beta_{\tau} \simeq 0.8 \times 10^{-6} \mathrm{~cm}^{2} / \mathrm{g}$ for the tau is rescaled to $\beta_{\tilde{\tau}, c} \simeq 9.5 \times 10^{-9}\left(150 \mathrm{GeV} / m_{\tilde{l}}\right)$ $\mathrm{cm}^{2} / \mathrm{g}$ according to the factor of $m_{\tau} / m_{\tilde{l}}$. With our new estimate of the stau range, parameterized by an energy dependent $\beta$, the effective range is increased relative to the range calculated using $\beta_{\tilde{\tau}, c}$.

Fig. 8 shows the ratio of the range using the energy dependent $\beta$ compared to the range calculated using the constant $\beta_{\tilde{\tau}, c}$, scaled appropriately for the different masses, for initial stau energies between $10^{4}-10^{12} \mathrm{GeV}$ and a minimum stau energy of $E_{0}=10^{3} \mathrm{GeV}$. The ratio can be as much as a factor of two for initial stau energies in the range of $10^{7}-10^{8} \mathrm{GeV}$. In this energy range, the $\beta$ term in Eq. (11) dominates over the ionization term $(\alpha)$ and the lifetime, but the effective $\beta$ is not as large as the rescaled constant $\beta_{\tilde{\tau}, c}$ in this energy range. The enhancement of the range by a factor of two is significant because it substantially improves the potential of neutrino telescopes for detecting evidence of stau production.

For $E_{0}=10^{6} \mathrm{GeV}$, a similar comparison between the Monte Carlo range and the range using $\beta_{\tilde{\tau}, c}$ is obtained. The ratio of the range determined by Monte Carlo propagation to the range evaluated with Eq. (11) using $\beta_{\tilde{\tau}, c}$ is a factor of $\sim 2$ for $E=10^{7}-10^{8} \mathrm{GeV}$ for $m_{\tilde{l}}=150 \mathrm{GeV}$ and a factor of about 1.6 for the same energy range with $m_{\tilde{l}}=250 \mathrm{GeV}$ for $\sqrt{F}=10^{7} \mathrm{GeV}$.

Estimates which scale the energy loss $\beta$ by $1 / m_{\tilde{l}}^{2}[21]$ dramatically overestimate the stau range at high ener- gies. Using the $\beta$ of Ref. [21], $\beta=3.9 \times 10^{-6}\left(m_{\mu} / m_{\tilde{l}}\right)^{2}$ $\mathrm{cm}^{2} / \mathrm{g}$, one finds that for an initial stau energy of $10^{6}$ $\mathrm{GeV}$, one has overestimated the range by about $40 \%$, and at $10^{7} \mathrm{GeV}$ by a factor of 4.8. The overestimate rapidly increases with energy. It is a factor of $\sim 30$ for an initial energy of $10^{9} \mathrm{GeV}$, a factor of $\sim 1600$ for an initial energy of $10^{12} \mathrm{GeV}$.

In summary, for the dominant photonuclear and $e^{+} e^{-}$ pair production processes, one expects qualitatively that the $\beta$ of a charged scalar particle (stau) scales as the inverse mass of the scalar particle. We have shown this result quantitatively by explicit calculation. Incorporating the electromagnetic energy loss of the stau and the long lifetime following from the supersymmetry breaking scale, we used a Monte Carlo to evaluate the range. The range determined by Monte Carlo evaluation is longer than the range of average energy loss using Eq. (11) and a constant $\beta_{\tilde{\tau}, c}$. Our energy dependent parametrization of an effective $\beta$ in Eq. (25) for the stau range reasonably approximates the Monte Carlo results. Our evaluation of the stau range suggests that previous estimates [1] of upward stau pair event rates, induced by supersymmetric particle production by upward-going neutrinos, may be enhanced by a factor of $\sim 2$ in some energy ranges.

\section{Acknowledgments}

This work was supported in part by DOE contracts DE-FG02-91ER40664, DE-FG02-04ER41319 and DEFG02-04ER41298 (Task C). We thank F. Halzen, S. Brodsky and M. Tannenbaum for helpful conversations.
[1] I. Albuquerque, G. Burdman and Z. Chacko, Phys. Rev. Lett. 92, 221802 (2004).

[2] M. Dine, W. Fischler, M. Srednicki, Nucl. Phys. B189, 575 (1981); S. Dimopoulos, S. Raby, Nucl. Phys. B192, 353 (1981); L. Alvarez-Gaumé, M. Claudson, M.B. Wise, Nucl. Phys. B207, 96, (1982); M. Dine, A.E. Nelson, Phys. Rev. D48, 1277 (1993); M. Dine, A.E. Nelson, Y. Shirman, Phys. Rev. D51, 1362 (1995); M. Dine, A.E. Nelson, Y. Nir, Y. Shirman, Phys. Rev. D53, 2658 (1996). For a review, see G.F. Giudice, R. Rattazzi, Phys. Rept. 332, 419 (1999).

[3] S. Eidelman et al. [Particle Data Group], Phys. Lett. B 592, 1 (2004).

[4] S. Iyer Dutta, M. H. Reno, I. Sarcevic and D. Seckel, Phys. Rev. D63, 094020 (2001).

[5] R. Devenish and A. Cooper-Sarkar, Deep Inelastic Scattering (Oxford University Press, New York, 2004).

[6] H. Abramowicz and A. Levy, hep-ph/9712415

[7] E. V. Bugaev and Y. V. Shlepin, Phys. Rev. D 67, 034027 (2003).

[8] B. Badelek, J. Kwiecinski and A. Stasto, Z. Phys. C 74, 297 (1997).

[9] M. J. Tannenbaum, Nucl. Instrum. Meth. A 300, 595 (1991).
[10] R. P. Kokoulin and A. A. Petrukhin, in Proc. of the XII International Conference on Cosmic Rays (Hobart, Tasmania, Australia, 1971), Vol. 6.

[11] V. B. Berestetskii, E. M. Lifshitz and L. P. Pitaevskii, Relativistic Quantum Theory, Part 1, translated by J. B. Sykes and J. S. Bell, (Pergamon Press, New York, 1971).

[12] L. Landau and E. M. Lifshitz, Physik Z. Sowjetunion 6, 244 (1934).

[13] See, e.g., S. Brodsky, T. Kinoshita and H. Terazawa, Phys. Rev. D 4, 1532 (1971).

[14] H. Meier, K. Hencken, D. Trautmann and G. Baur, Eur. Phys. J. C 2, 741 (1998).

[15] S. R. Kelner, Sov. J. Nucl. Phys. 5, 778 (1967).

[16] G. P. Lepage, J. Comp. Phys. 27, 192 (1978).

[17] D. Ivanov et al., Phys. Lett. B442, 453 (1998).

[18] See, e.g., O. Nachtmann, Elementary Particle Physics: Concepts and Phenomena (Springer-Verlag, New York, 1990).

[19] A. A. Petrukhin and V. V. Shestakov, Can. J. Phys. 46, S377 (1968).

[20] P. Lipari and T. Stanev, Phys. Rev. D 44, 3543 (1991).

[21] X. J. Bi, J. X. Wang, C. Zhang and X. M. Zhang, Phys. Rev. D 70, 123512 (2004). 